\title{
A Comparative Investigation of an in vitro and Clinical Test of the Bifidogenic Effect of an Infant Formula
}

\author{
Nikoleta M. Lugonja, ${ }^{1 *}$, Olga B. Martinov², Mirjana R. Rasovic ${ }^{3}$, Snezana D. Spasic ${ }^{2}$, \\ Gordana Dj. Gojgic ${ }^{2}$ and Miroslav M. Vrvic ${ }^{1,2}$ \\ ${ }^{1}$ Faculty of Chemistry, University of Belgrade, 11158 Belgrade, Studentski trg 16, P.O. Box 51, Serbia \\ ${ }^{2}$ Department of Chemistry, Institute of Chemistry, Technology and Metallurgy, 11001 Belgrade, Njegoseva 12, P.O. \\ Box 473, Serbia \\ ${ }^{3}$ Center for the Welfare of Infants, Children and Adolescents, Zvecanska 7, 11001 Belgrade, Serbia
}

Received 13 May, 2010; Accepted 28 June, 2010; Published online 29 September, 2010

\begin{abstract}
Summary The bifidogenic effect of an infant formula supplemented with inulin and fructooligosaccharides $(4.0 \mathrm{~g} / \mathrm{l})$ was examined clinically and in vitro, and compared that of mature breast milk. In a 28-day clinical study, fecal samples of 21 infants, divided into two groups: one receiving the infant formula and the other breast milk, were microbiologically and biochemically examined. In the in vitro investigation, microbiological and biochemical changes in the infant formula and breast milk induced by the action of bifidobacteria isolated from infant feces were examined. There were no significant differences in the fecal numbers of lactobacilli, total aerobes, anaerobes or yeasts and fungi. In contrast, the bifidobacteria numbers in the stools increased significantly during the study in the infants receiving the supplemented formula. The comparative in vitro test showed that the bifidogenic effect was similar for infant formula and breast milk in terms of the number of bifidobacteria. Consumption of infant formula with added inulin and fructooligosaccharides stimulated the bifidogenic effect, both clinically and in vitro. The in vitro test can quickly and objectively determine the bifidogenic effect of infant formula and indicate their quality. However, a clinical test is necessary to determine the acceptance and biological value of infant formula.
\end{abstract}

Key Words: infant formula, inulin, clinical examination, in vitro test, bifidogenic effect

\section{Introduction}

During birth and the days following, the sterile gastrointestinal tract of newborn infants becomes inoculated and colonized with bacteria derived from the mother and the environment $[1,2]$. Different factors, including the type of birth (vaginal or caesarean), the microflora of the mother's vagina and skin, hygiene during birth, the newborn's environment, antibiotic regime and, above all, the type of diet, influence the development of the gastrointestinal tract [3].

\footnotetext{
*To whom correspondence should be addressed.

Tel: +381112637273 Fax: +381112636061

E-mail: nikoleta@chem.bg.ac.rs
}

Human breast milk is the best dietary choice for newborn infants, as it protects unweaned infants from allergies, infections and oxidative stress; breastfeeding also contributes to a closer relationship between mother and child [4-7]. Milk composition varies during the first days after birth and then becomes suitable for the satisfaction of the dietary requirements of infants.

Breast milk is considered the gold standard against which the quality of adapted formula milks is assessed. Formula feeds, used by women who cannot breastfeed, should satisfy all the nutritional requirements of the unweaned infant and allow normal development. The intestinal microflora of unweaned infants fed on formulas is more diverse than that of infants fed on breast milk, and contains predominantly bifidobacteria, and also significant quantities of Entero- 
bacteriaceae and gram-negative bacteria [8-10]. Bifidobacteria and lactobacilli are known to produce lactic, acetic and other organic acids, which reduce the $\mathrm{pH}$ in the colon and inhibit the growth of some pathogenic microorganisms $[11,12]$.

The intestinal microflora of newborn infants can be modified by the intake of dietary components, such as probiotics or prebiotics. Prebiotics are indigestible compounds found in foods which selectively stimulate the growth and metabolic activity of the "good" bacterial species Bifidobacterium and Lactobacillus in the colon and improve the health of the host $[13,14]$. Oligosaccharides and other components of breast milk are likely to be important sources of the "prebiotic" effect. These indigestible oligosaccharides in breast milk act on the microflora and, particularly, contribute to the development of the predominating colonic bifidobacterial microflora in newborn infants $[15,16]$. Fructans in the concentration range of $2-10 \mathrm{~g} / 1$ are considered safe for use in the diets of children and unweaned infants [16]. Inulin, an indigestible polysaccharide, is a natural prebiotic which produces many desirable effects in humans, including increased production of short-chain fatty acids, stimulation of the growth and activity of bifidobacteria, reduced serum cholesterol and triglycerides, and reduced risks of colon cancer and colonic and intestinal infections [17-20]. The examination of inulin-type prebiotics and their applications are undergoing continual development due to increasingly efficacious new molecular assessment methods $[21,22]$.

The aim of this study was to investigate the influence of an infant formula containing added inulin and fructooligosaccharides (FOS) on the gut microflora, as well as on the growth and development of infants compared with infants who were exclusively breastfed (considered the gold standard for infant diets). The abilities of breast milk and the infant formula to undergo bifidogenesis, a microbiological indicator of the bifidogenic effect, were compared in a clinical study and in an in vitro test. Infant growth and development as well as the intestinal tolerance of the newborn infants fed on the formula were followed. Finally, the usefulness of the in vitro test as a rapid indicator of the bifidigenic effect of formula feeds, compared to a clinical study was evaluated.

\section{Materials and Methods}

\section{Study population}

Twenty one healthy, full-term, vaginally born infants with a normal birth weight were enrolled in the study via the Center for the Welfare of Infants, Children and Adolescents, in Belgrade. All babies were younger than six months (age between 5 and 16 weeks). The exclusion criteria were as follows: antibiotic treatments, diarrhea, iron supplementa- tion and intake of other prebiotics or probiotics. Mothers of breast-fed infants were required to be in good health and not to take antibiotics. The protocol and procedures for this clinical study were fully approved by the Board of the Center for the Welfare of Infants, Children and Adolescents.

\section{Diet and experimental design}

The 28-day study enrolled 21 infants of both sexes. During the study period, infants were divided into two groups: one group of infants ( 6 boys and 5 girls) was fully formula-fed (FF), while the control group ( 7 boys and 3 girls) were exclusively breast milk-fed (BMF) by their mothers. The FF group received an experimental full-term infant formula designed for infants less than 6 months of age and supplemented with inulin and FOS (4.0 g/l). The inulin and FOS used in this study were produced from natural chicory. The infant milk-based formula consisted of: lipids (26.9), proteins (10.7) and carbohydrates (58.6) expressed as $\mathrm{g} / 100 \mathrm{~g}$ of powder, plus smaller amounts of vitamins (A, C, $\mathrm{D}$ and $\mathrm{E})$ and microelements. The main ingredients were skimmed milk, lactalbumin, demineralized whey, lactose, maltodextrin, glucose syrup, taurine, sunflower oils and soybean lecithin.

The BMF and FF infants had the same schedule of visits and specimen collection. Every day during the study, the weight and length of the infants, number of feeds and tolerance to the formula intake, and stool frequency and consistency were recorded. The stool frequency was reported as the number of stools in the previous $24 \mathrm{~h}$ period and the consistency as soft, normal or hard.

Stools for bacteriological and biochemical analysis were collected three times during the 28-day study: D0 (D-day), before the observation period; D14, in the middle of the supplementation period; and D28 at the end of the study. After collection in sterile containers, the stools were immediately placed at $4^{\circ} \mathrm{C}$ and transferred to the laboratory for analysis.

\section{Tolerance and well-being}

The gastrointestinal symptoms and general health information for each infant were evaluated daily by their parents and care-givers from the care center. Slight gastrointestinal disorders, decreased appetite, flatus and regurgitations were recorded daily, and were considered as signs of discomfort, but not as signs to suspend the study.

\section{Laboratory methods}

All fecal analyses, microbiological and biochemical, were performed on fresh stools, immediately after transfer to the laboratory.

\section{Microbiological analysis of the fecal samples}

Freshly collected fecal samples were well mixed with 
physiological saline $(1 \mathrm{~g}$ in $99 \mathrm{ml})$ and serially diluted in the same solution. Appropriate dilutions $(1 \mathrm{ml})$ were transferred to sterile Petri dishes and immediately pour-plated to isolate and quantify bifidobacteria, lactobacilli, aerobes, anaerobes and yeasts and fungi. Selective Bifidobacteria Medium with Lactulose (BML) [23] was used for the isolation of bifidobacteria. Lactobacilli were enumerated using Rogosa agar [24]. Nutrient agar was employed for the enumeration of total aerobic and facultative anaerobic bacteria. Sabuorauddextrose agar was used for the enumeration of the total yeasts and fungi. Nutrient agar with $0.5 \%$ glucose was used for enumeration of the total anaerobic bacteria. Bifidobacteria and anaerobic bacteria were incubated under anaerobic conditions (5\% carbon dioxide $\left(\mathrm{CO}_{2}\right)$ ) in an anaerostat (Heraeus, Hanau, Germany) at $37^{\circ} \mathrm{C}$ for $48 \mathrm{~h}$. Lactobacilli and aerobic bacteria were incubated aerobically at $37^{\circ} \mathrm{C}$ for $48 \mathrm{~h}$. Yeasts and fungi were incubated aerobically at $28^{\circ} \mathrm{C}$ for $48 \mathrm{~h}$. Bacterial, yeast and fungal counts were expressed as $\mathrm{CFU} / \mathrm{g}$ of feces.

Bacteria were identified using routine laboratory methods. Bifidobacterium spp. were identified according their morphology and the Rapid ID 32 ${ }^{\circledR}$ identification system for anaerobes (BioMerieux, Marcy-l'Etoile, France).

\section{Biochemical analysis of the fecal samples}

The fecal $\mathrm{pH}$ was measured using a $\mathrm{pH}$-meter, type PHM 26, with a combined glass electrode, type GK 2401C (Radiometer). Total organic acids (TOA) were determined by automatic potentiometric titration (titrator type TT 2, Tacussel, France, combined glass electrode, type GK 2401C, Radiometer) of $50 \mathrm{ml}$ samples with a standard $0.1 \mathrm{M}$ $\mathrm{NaOH}$ solution. The results were calculated as lactic acid equivalents and are expressed as g/100 $\mathrm{ml}$. Stool water content in feces, computed as \% per $g$ of feces, was determined using a hygrometer (Shimazu-moisture balance MOC-120H). All chemicals employed in this study were of analytical grade and purchased from Merck (Darmstadt, Germany), Sigma (Steinheim, Germany) and Torlak (Belgrade, Serbia).

Material for the isolation of bifidobacteria for the in vitro test and the preparation of the inoculum

Material for the isolation of the accumulated mixed culture of Bifidobacterium spp. was $1 \mathrm{~g}$ of a mixture of the 30 feces of healthy, vaginally and full-term born babies, who were exclusively nursed and who did not consume antibiotics. The feces were sampled three days after birth, directly upon excretion. All samples were frozen immediately at $-20^{\circ} \mathrm{C}$ until the preparation of the inoculum.

Bifidobacteria were isolated on a solid selective BML. The inoculum was prepared from the third $48 \mathrm{~h}$ subculture on a BML agar slant, into $10 \mathrm{ml}$ liquid BML, which was then incubated for $48 \mathrm{~h}$ at $37^{\circ} \mathrm{C}$ in an anaerostat (Heraeus) in $5 \%$
$\mathrm{CO}_{2}$. The total volume required was prepared by mixing several individual inoculums in order to obtain a large enough biomass for all the required seedings.

\section{Substrate for the in vitro test}

Experimental full-term infant formula milk supplemented with inulin and FOS $(4.0 \mathrm{~g} / \mathrm{l})$ was used as infant food $(100 \mathrm{ml}$ ready meal was prepared from $14 \mathrm{~g}$ of powder and $90 \mathrm{ml}$ of water; designation IF). Mature breast milk (designation MBM) was the reference substrate and it was comprised of equal volumes of 20 breast milks obtained from 20 healthy mothers who had delivered full-term infants (the samples were obtained from 3 to 10 days postpartum).

\section{Pancreatic digestion-digestion simulation [25]}

Digestion was realized in $300 \mathrm{ml}$ wide-necked Erlenmeyer flasks containing $100 \mathrm{ml}$ of substrate plus 50,000 USNF units [26] each of amylase and protease and 4,000 USNF units of lipase (pancreatin powder; A. Costantino \& C. Favria, Italy). The process was performed by immersion in a water bath at $37^{\circ} \mathrm{C}$ for $2 \mathrm{~h}$, with occasional shaking. Digestion was halted by sterilization $\left(121^{\circ} \mathrm{C}\right.$ for $\left.20 \mathrm{~min}\right)$, which induced thermal deactivation of the enzymes. For one series of experiments, two Erlenmeyer flasks with the same substrates were prepared, one of which was used after inoculation for the determination of the parameters at the beginning of the test.

\section{In vitro bifidogenesis}

Sterile "digested" substrates were inoculated with $1.0 \mathrm{ml}$ of inoculum from the same accumulated mixed culture of bifidobacteria. Bifidogenesis lasted $48 \mathrm{~h}$ at $37^{\circ} \mathrm{C}$ in an anaerostat with $5 \% \mathrm{CO}_{2}$.

\section{Identification of the bifidobacterial isolates in vitro}

From the BML medium, colonies were picked and identified as a member of the genus Bifidobacterium by typical morphology and RAPID ID $32 \mathrm{~A}^{\circledR}$ test results. To examine the morphology, a native preparation was prepared from the substrate and observed with a light microscope operating in the bright field (Laboval, Zeiss, Germany).

\section{Determination of the total number of in vitro bifidobacteria (TNB) [27]}

Suitable dilutions of substrates and pure cultures of bifidobacteria were prepared in physiological saline and spread-plated onto BML agar. The BML agar plates were incubated for $48 \mathrm{~h}$ at $37^{\circ} \mathrm{C}$ in a $5 \% \mathrm{CO}_{2}$ atmosphere, after which the isolated bifidobacteria colonies on the plates were counted to obtain the TNB (expressed as colony-forming units per $\mathrm{ml}(\mathrm{CFU} / \mathrm{ml}))$. 


\section{Biochemical analysis of the in vitro milk samples}

The dry biomass (DBM; g/100 ml) [28] was determined gravimetrically from $10 \mathrm{ml}$ culture-inoculated substrate, by centrifugation at 4,500 rpm for $10 \mathrm{~min}$, triple water rinsing and measurement using a hygrometer (Shimazu, Kyoto, Japan). The $\mathrm{pH}$ and total organic acids were determined as described before. The molar ratio of acetic and lactic acid $(M R A L)$ was determined by enzymatic tests in accordance with the producer's instructions (Randox, UK) [29, 30], modified for these substrates (filtration through a $0.2 \mu \mathrm{m}$ pore size membrane and without alkaline hydrolysis).

\section{Statistical analysis}

All statistical analyses were realized using the statistical software package SPSS for Windows V. 11.5. Values are reported as means \pm standard deviation (SD). Values of $p<0.05$ were considered statistically significant. Parameters (anthropometric, microbiological and biochemical) were tested for normal distribution and compared using the $t$ test. Analysis of the influence of the type of feeding was performed by multivariate analysis of variance with repeated measurements. Internal Group Factor (within subject factors - F1) and Factor of Differences between the Groups (between subject factors $-F 1$ group) were used to test for significant differences between the study period and mode of feeding.

\section{Results}

A clinical study and an in vitro artificial model simulating gastrointestinal transit were used to compare the bifidogenic effect of an infant formula with added inulin and FOS with that of breast milk. The clinical study compared the effects on healthy infants of feeding the supplemented infant formula compared to breast milk. The in vitro test consisted of successive incubation chambers containing either IF or MBM, artificially digested and subsequently inoculated and incubated with bifidobacteria.

In the clinical study, the study groups were comparable with respect to clinical characteristics (Table 1). At the beginning of the study, the ages of the infants were similar; $8.0 \pm 2.6$ and $9.3 \pm 3.3$ weeks for the FF and BMF groups, respectively. The body weight and length of the infants in both study groups increased at similar rates during the study period and were within the normal framework for the postnatal period. All infants exhibited normal growth during the study.

\section{Tolerance and well-being}

In the study, all infants in each group liked the offered milks. The intake of the bottle formula with added inulin and FOS at $4.0 \mathrm{~g} / \mathrm{l}$ was well tolerated by the infants: the incidence of side effects did not differ among the feeding

Table 1. Clinical data in groups of the infants enrolled in the study (given as mean \pm SD)

\begin{tabular}{|c|c|c|c|}
\hline \multirow[b]{2}{*}{ Type of feeding } & \multicolumn{2}{|c|}{ Study group } & \multirow[b]{2}{*}{$p^{*}$} \\
\hline & $\begin{array}{l}\text { Infant formula with added inulin } \\
\text { and FOS (FF) }\end{array}$ & Breastfeeding (BMF) & \\
\hline Number of infants $(\mathrm{M} / \mathrm{F})$ & $11(6 / 5)$ & $10(7 / 3)$ & 0.695 \\
\hline Postnatal age (weeks) & $8.0 \pm 2.6$ & $9.3 \pm 3.3$ & 0.327 \\
\hline \multicolumn{4}{|l|}{ Weight (g) } \\
\hline D0 & $4124.55 \pm 662.40$ & $4838.0 \pm 815.58$ & 0.040 \\
\hline D28 & $4800.91 \pm 675.76$ & $5453.0 \pm 809.73$ & 0.059 \\
\hline Weight Gain & $676.36 \pm 294.11$ & $615.0 \pm 243.50$ & 0.611 \\
\hline \multicolumn{4}{|l|}{ Length $(\mathrm{cm})$} \\
\hline D0 & $54.36 \pm 3.47$ & $55.80 \pm 3.58$ & 0.363 \\
\hline D28 & $57.73 \pm 3.74$ & $59.20 \pm 3.43$ & 0.360 \\
\hline Length Gain & $3.37 \pm 2.60$ & $3.40 \pm 0.84$ & 0.967 \\
\hline \multicolumn{4}{|l|}{ Feeding } \\
\hline No. of feeding (per day) & $6.36 \pm 0.50$ & $7.10 \pm 0.57$ & 0.005 \\
\hline Acceptance (Yes/No) & Yes & Yes & - \\
\hline \multicolumn{4}{|l|}{ Stool } \\
\hline Number (per day) & $3.18 \pm 0.40$ & $2.50 \pm 0.71$ & 0.051 \\
\hline Consistency & Normal $72.7 \%$ & Normal $60 \%$ & 0.659 \\
\hline & Soft $27.32 \%$ & Soft $40 \%$ & \\
\hline
\end{tabular}

*The $p$ value represents the significance level of the difference in the change between the groups during the study.

**Weight and length gain were calculated for the time period between the start and end of the study. Age at the beginning of the study is given in weeks.

Vol. 47, No. 3, 2010 
groups during the study. Stool frequency and consistency, and side effects (flatus and regurgitations) were similar between the groups.

\section{Gut microbiota}

Modifications in the levels of microbial colonization between the beginning D0, D14 and the end of the supplementation for both groups are compared in Table 2 . The bacterial counts are expressed as means $\pm \mathrm{SD} 10^{8} \mathrm{CFU} / \mathrm{g}$ feces.

At D0 and D14, there were no significant differences in bifidobacteria counts in the stools of FF group and the BMF control group. By D28, however, the bifidobacterial levels in the stools from the FF infants was significantly higher than in the BMF infants $\left(p^{28}=0.008\right)$. During the study, the bifidobacterial counts increased statistically significantly in both groups, but the dynamics of the change in the $F 1$ group differed between groups: the mean bifidobacteria level in the stools from the FF infants continually rose, from 11.42 (D0), through 30.26 (D14) to 89.45 (D28) $10^{8} \mathrm{CFU} / \mathrm{g}$ feces, while in the BMF group, the levels increased at the beginning of the study (from 8.83 at D0 to 17.56 by D14) but then remained until D28 $\left(17.53 \times 10^{8} \mathrm{CFU} / \mathrm{g}\right.$ feces $)$.

The number of lactobacilli, as intestinal probiotics, increased in both the FF and BMF infants, without statistically significant differences in the numbers or dynamic of change during the study. Simultaneously with the increase in bifidobacterial and lactobacilli populations, decreases in the total aerobes, anaerobes and yeasts and fungi levels were observed. During the study, the numbers of total aerobes and yeasts and fungi continually decreased in the stools of the FF infants, without statistically significant differences between the groups at the end of the study. The anaerobes decreased in both groups during the study, with significant differences with time but without significantly different rates of change.

\section{Biochemical parameters}

The type of feed affected the $\mathrm{pH}$ value and TOA content of the stools obtained from the infants during the study (Table 3). The FF and BMF groups were statistically different in the dynamics of the change $\mathrm{pH}$ in the groups $(p<0.05)$. Stool $\mathrm{pH}$ continually decreased in the FF group but in the BMF group, the $\mathrm{pH}$ decreased from D0 to D14 of study and thereafter slightly increased to the end of the

Table 2. Level of infant's gut colonization in the FF and BMF groups (given as mean \pm SD)

\begin{tabular}{|c|c|c|c|c|c|}
\hline \multirow[b]{2}{*}{ Type of feeding } & \multicolumn{2}{|c|}{$\mathrm{CFU} \times 10^{8} / \mathrm{g}$ of feces in the following group } & \multirow[b]{2}{*}{$p^{*}$} & \multirow[b]{2}{*}{$F 1^{* *}$} & \multirow[b]{2}{*}{ F1 group** } \\
\hline & $\begin{array}{c}\text { Infant formula with added } \\
\text { inulin and FOS (FF) }\end{array}$ & Breastfeeding (BMF) & & & \\
\hline Bifidobacterium & & & & 0.006 & 0.024 \\
\hline D0 & $11.42 \pm 10.72$ & $8.83 \pm 9.62$ & 0.567 & & \\
\hline D14 & $30.26 \pm 21.82$ & $17.56 \pm 32.86$ & 0.305 & & \\
\hline D28 & $89.45 \pm 73.62$ & $17.53 \pm 21.47$ & 0.008 & & \\
\hline Lactobacillus & & & & 0.134 & 0.349 \\
\hline D0 & $17.22 \pm 9.67$ & $17.47 \pm 16.31$ & 0.965 & & \\
\hline D14 & $64.64 \pm 77.38$ & $26.77 \pm 41.11$ & 0.184 & & \\
\hline D28 & $137.99 \pm 227.78$ & $33.48 \pm 47.42$ & 0.172 & & \\
\hline Aerobes & & & & 0.135 & 0.107 \\
\hline D0 & $86.11 \pm 117.96$ & $42.86 \pm 97.89$ & 0.375 & & \\
\hline D14 & $32.80 \pm 21.34$ & $19.72 \pm 18.72$ & 0.154 & & \\
\hline D28 & $14.46 \pm 19.92$ & $22.30 \pm 29.21$ & 0.477 & & \\
\hline Anaerobes & & & & 0.040 & 0.987 \\
\hline D0 & $98.37 \pm 80.72$ & $117.04 \pm 184.36$ & 0.763 & & \\
\hline D14 & $75.89 \pm 101.44$ & $83.60 \pm 162.06$ & 0.896 & & \\
\hline D28 & $26.31 \pm 25.09$ & $42.94 \pm 7.09$ & 0.160 & & \\
\hline Yeasts and Fungi & & & & 0.296 & 0.075 \\
\hline D0 & $25.78 \pm 35.20$ & $8.68 \pm 14.03$ & 0.168 & & \\
\hline D14 & $13.22 \pm 9.34$ & $3.67 \pm 3.73$ & 0.007 & & \\
\hline D28 & $5.06 \pm 5.68$ & $9.32 \pm 16.09$ & 0.419 & & \\
\hline
\end{tabular}

*The $p$ value represents the significance level of the difference in the change between the groups during the study, determined by the independent-sample $t$ test.

**Fl and $F 1$ group - given as the internal group factor (within subject factors $-F 1$ ) and as the factor of differences between the groups (between subject factors-Fl group). 
Table 3. Biochemical data in stools from the groups of the infants enrolled in the study (given as mean \pm SD)

\begin{tabular}{|c|c|c|c|c|c|}
\hline \multirow[b]{2}{*}{ Type of feeding } & \multicolumn{2}{|c|}{ Study group } & \multirow[b]{2}{*}{$p^{*}$} & \multirow[b]{2}{*}{$F 1^{* *}$} & \multirow[b]{2}{*}{ F1 group** } \\
\hline & $\begin{array}{c}\text { Infant formula with added } \\
\text { inulin and FOS (FF) }\end{array}$ & Breastfeeding (BMF) & & & \\
\hline $\mathrm{pH}$ & & & & 0.001 & 0.001 \\
\hline D0 & $7.03 \pm 0.83$ & $6.11 \pm 1.32$ & 0.070 & & \\
\hline D14 & $6.52 \pm 0.44$ & $5.95 \pm 1.15$ & 0.146 & & \\
\hline D28 & $5.90 \pm 0.35$ & $6.01 \pm 1.39$ & 0.798 & & \\
\hline TOA & & & & 0.003 & 0.027 \\
\hline D0 & $0.0156 \pm 0.0226$ & $0.0221 \pm 0.0295$ & 0.577 & & \\
\hline D14 & $0.0199 \pm 0.0176$ & $0.0288 \pm 0.0253$ & 0.356 & & \\
\hline D28 & $0.0401 \pm 0.0218$ & $0.0288 \pm 0.0236$ & 0.151 & & \\
\hline Water content $(\%)$ & & & & 0.038 & 0.140 \\
\hline D0 & $73.87 \pm 6.52$ & $77.60 \pm 8.74$ & 0.280 & & \\
\hline D14 & $76.49 \pm 4.62$ & $69.62 \pm 19.68$ & 0.274 & & \\
\hline D28 & $77.93 \pm 4.70$ & $81.18 \pm 5.27$ & 0.151 & & \\
\hline
\end{tabular}

*The $p$ value represents the significance level of the difference in the change between the groups during the study.

${ }^{* *} F 1$ and $F 1$ group - given as the internal group factor (within subject factors $-F 1$ ) and as the factor of differences between the groups (between subject factors- $F 1$ group).

TOA-Total organic acids, calculated as lactic acid $(\mathrm{g} / 100 \mathrm{ml})$.

study. The TOA in the stools from both groups, calculated as lactic acid, increased during time but at the end of the study was higher in the FF than in the BMF infants. The influence of the diet on the change in fecal $\mathrm{pH}$ and TOA was statistically significant $(p<0.05)$.

The feed type significantly influenced the stool consistency scores, computed as $\%$ water content in the stool sample. For the FF infants, the consistency changed to softer stools, close to the scores found for the BMF infants. This confirms the anthropometric results for stool consistency, where $72 \%$ of stools from the FF infants were normal consistency vs $60 \%$ of those from the BMF infants.

\section{In vitro test}

Microbiological and biochemical indicators were determined at the beginning and at the end of the in vitro test (Table 4). Microbiological parameters for both substrates (IF and MBM) were identical at the beginning of the test (TNB, $6.27 \times 10^{8} \mathrm{CFU} / \mathrm{ml}$ and $D B M, 0.029 \mathrm{~g} / 100 \mathrm{ml}$ ). In the native preparation, the bifidobacterial cells were extremely small and of irregular, characteristically elongated shape. After $48 \mathrm{~h}$, the $T N B$ in both substrates had increased and was higher in the IF $\left(1.12 \times 10^{10} / \mathrm{ml}\right)$ than in MBM group $\left(4.9 \times 10^{9} / \mathrm{ml}\right)$. As was to be expected, the most suitable substrate for the production of biomass was MBM $(0.262$ $\mathrm{g} / 100 \mathrm{ml})$ compared with IF $(0.234 \mathrm{~g} / 100 \mathrm{ml})$, after $48 \mathrm{~h}$ of bifidogenesis. The bifidogenic index $(B I)$ is the ratio of bifidobacteria dry biomass obtained at the end of the test with infant formula divided by that obtained with breast milk in the same series of the experiment multiplied by
Table 4. Microbiological and biochemical indicators of in vitro bifidogenesis (given as mean $\pm \mathrm{SD}$ )

\begin{tabular}{ccc}
\hline Substrate & $\begin{array}{c}\text { Infant formula with added } \\
\text { inulin and FOS (IF) }\end{array}$ & $\begin{array}{c}\text { Mature breast milk } \\
(\mathrm{MBM})\end{array}$ \\
\hline$T N B$ & & \\
$T N B_{0}$ & $6.27 \pm 0.59$ & $6.27 \pm 0.59$ \\
$T N B_{48}$ & $1.12 \pm 0.22$ & $0.49 \pm 0.38$ \\
$D B M$ & & \\
$D B M_{0}$ & $0.029 \pm 0.012$ & $0.029 \pm 0.012$ \\
$D B M_{48}$ & $0.234 \pm 0.044$ & $0.262 \pm 0.036$ \\
$\mathrm{pH}$ & & \\
$\mathrm{pH}$ & 6.73 & 7.04 \\
$\mathrm{pH} 0$ & 5.40 & 5.79 \\
$\mathrm{pH} 48$ & 4.32 & 4.94 \\
$T O A$ & 0.145 & 0.067 \\
$M R A L$ & $3.06: 1.86$ & $2.95: 1.90$ \\
$B I(\%)$ & 0.89 & 1 \\
\hline
\end{tabular}

$\mathrm{TNB}_{0}$ - Total number of bifidobacteria expressed as CFU $\times 10^{8} / \mathrm{ml}$ (at the beginning).

$T N B_{48}$ - Total number of bifidobacteria expressed as CFU $\times 10^{10} / \mathrm{ml}$ (at the end).

$D B M$-Dry biomass $(\mathrm{g} / 100 \mathrm{ml})$.

$\mathrm{pH}^{\mathrm{a}}$ after pancreatic enzymes-simulated digestion.

$\mathrm{pH}_{0}$ at the beginning of the experiment.

$\mathrm{pH}_{48}$ at the end of the test.

TOA - Total organic acids, calculated as lactic acid $(\mathrm{g} / 100 \mathrm{ml})$.

$M R A L$ - Mole ratio of acetic and lactic acid.

$B I$-Bifidogenic index. 
hundred (i.e., expressed as a percentage); it is a discriminatory criterion for an estimation of the bifidogenic effects. The $B I$ values obtained in this study showed that IF had a high $B I$ value (0.89).

At the beginning of the in vitro test, the TOA values were $0.005 \mathrm{~g} / 100 \mathrm{ml}$ in both substrates. At the end of the process, the concentration of the bacterially generated organic acids was higher in the IF $(0.145 \mathrm{~g} / 100 \mathrm{ml})$ than in the reference substrate, MBM $(0.067 \mathrm{~g} / 100 \mathrm{ml})$, while the substrate $\mathrm{pH}$ values were 4.32 and 4.94 for IF and MBM, respectively. The mole ratio of the produced acetic and lactic acids in both tested substrates was very close to $3: 2$, which is a physiological and biochemical characteristic of the genus Bifidobacterium [31].

\section{Discussion}

Studies published to date have shown that interactions exist between infant's dietary types, intestinal flora and the development of the gastrointestinal tract and the immune system [20]. As a result of these complex interactions, dietary factors, including prebiotics, can improve the growth and function of intestinal bacteria [1]. This study showed both clinically and in vitro that inulin and FOS, as prebiotic additives in infant formula, can influence bifidogenesis in the infant. From the results of the in vitro test and clinical investigation, which studied bifidogenesis in unweaned infants younger than 6 months fed on an infant formula containing additional inulin and FOS or breastfed, the following can be concluded: The total numbers of bifidobacteria observed in the clinical study were greater in the stools from FF than in the stools from BMF infants; bifidobacteria were also observed in an in vitro test, the IF substrate contained more bifidobacteria than MBM-the gold standard. The dry biomass of bifidobacteria, i.e., the bifidogenic effect, which is one of the most important parameters for assessing the biological value and overall quality of infant formula, is the best indicator for comparing these products. The bifidogenic index, established in the in vitro test as a discriminatory criterion for describing the bifidogenic effect, showed the relationship between dry biomass in the examined sample compared with that of breast milk, and clearly categorized the tested product. The examined infant formula stimulated the growth of bifidobacteria to a high degree; in fact, to a level very similar to that achieved by human breast milk. A healthy microflora is essential for the general development of unweaned infants, since it plays an important role in the normal diet and metabolism. The clinical study showed that the infant formula supplemented with inulin and FOS stimulated the growth of bifidobacteria in the intestine, resulting in a reduced $\mathrm{pH}$ and increased content of total organic acids; this was also found in the in vitro test. The increase of the
TOA value in combination with the lowered $\mathrm{pH}$ value influences the growth of other groups of bacteria, as well as yeasts and fungi in the colon, since their growth is inhibited by a combination of weak organic acids and low $\mathrm{pH}$, as well as by specific bacteriocins secreted by lactic acid bacteria $[32,33]$. The changes induced by the prebiotics inulin and FOS contribute to the intestinal flora and their metabolic activity in unweaned infants fed with these compounds in a manner very similar to that of infants exclusively nursed on breast milk.

The biochemical and microbiological indicators in the in vitro test resulted from changes in the substrates, which were exclusively caused by the action of bifidobacteria, and stimulation of their proliferation by the prebiotics in the infant formula and in breast milk.

The molar ratio of acetic to lactic acids in both in vitrotested milks was close to $3: 2$, which is a physiological and biochemical characteristic of the genus Bifidobacterium (with no production of $\mathrm{CO}_{2}$ ) [31]. This result additionally confirms, besides the RAPID ID $32 \mathrm{~A}^{\circledR}$ system for identification of anaerobes, that the isolated accumulated mixed culture used to inoculate the in vitro test was comprised only of bifidobacterial species and that changes in the substrates were the result of their actions and bifidogenesis. This, therefore, is a control indicator that the changes really were the consequence of the bifidogenesis effect in the substrates.

The clinical test of the infant formula containing additional inulin and FOS showed that a diet with this formula had no statistically significant effects on the anthropometric factors of growth and development of the infants (length, weight) compared with the control group of breastfed infants. In addition, the development of the intestinal flora, which (especially the bifidobacteria) is less developed in children fed exclusively on adapted formula [34], was at the level of breast milk with the examined formula. The numbers of microorganisms found in the FF group were statistically similar to those found in the control group, except for bifidobacteria, which were present in significantly greater numbers. It is important that the number of bifidobacteria in infants feeding on formula is as high as possible, since these organisms protect the infants from allergies and infections $[4,5]$. The number and consistency of stools did not statistically differ between the two groups of infants, which is important since FF infants often pass firmer stools than breastfed infants [35].

Based on the results obtained during the clinical and in vitro tests conducted during this study, it can be concluded that the infant formula containing additional prebiotics (inulin and FOS) and mature breast milk have similar bifidogenic effects. Infant formula containing additional inulin and FOS obtained from natural sources is safe for use, it favorably influences the growth and development of infants, is well accepted, and favorably influences the 
development of good microflora and stool consistency.

The artificial system developed to estimate the bifidogenic effect in vitro can be used to rapidly and objectively determine the bifidogenic effect of infant formula compared to mature breast milk as a reference substrate. Under controlled conditions, the in vitro test is a rapid method for the determination of the bifidogenic effect, as it takes just $48 \mathrm{~h}$. Microbiological and biochemical indicators determined in the test are suitable for following the process of in vitro bifidogenesis. The dry biomass of bifidobacteria is the best indicator, the use of which enables the bifidogenic effects of infant formula to be compared, and it is one of the most important parameters of the biological value and overall quality of these foods. The Bifidogenic Index conducted as a discriminatory criterion for determining the bifidogenic effect, clearly categorizes and ranks the tested products.

The in vitro test described in this study allows the bifidogenic effect of infant formula to be rapidly and objectively confirmed in a controlled manner, in contrast to a clinical study, which lasts several months and depends on a large number of factors, many of which cannot be influenced or controlled. However, this in vitro test cannot reproduce the natural conditions in the gut of unweaned infants. Therefore, it is also necessary to clinically test new formula, and determine their acceptability and biological value. For these reasons, this in vitro test should be used in the first phase for confirmation of the bifidogenic effect. It can also be employed as a discriminatory test to compare and rank different infant formula.

\section{Acknowledgments}

This research was supported in part by Grant No. 142018B from the Ministry of Science and Technological Development of the Republic of Serbia. We thank Dragana Cveticanin, MD, and the staff of the Center for the Welfare of Infants, Children and Adolescents, Belgrade, for their cooperation in this study. We also thank all the families who provided the fecal samples.

\section{Abbreviations}

FOS, Fructooligosaccharides; FF, Formula-fed; BMF, Breast milk-fed; D, Day; BML, Selective Medium with Lactulose; TOA, Total organic acids, calculated as lactic acid; IF, Experimental full-term infant formula milk; MBM, Breast milk; TNB, Total number of bifidobacteria; CFU, Colony forming units; $D B M$, Dry biomass; $M R A L$, Molar ratio of acetic and lactic acid; $S D$, Standard deviation; $F 1$, Within subject factor; $F 1$ group, Between subjects factor; $B I$, Bifidogenic Index.

\section{References}

[1] Fanaro, S., Chierici, R., Guerrini, P., and Vigi, V.: Intestinal microflora in early infancy: composition and development. Acta Paediatr. Suppl., 91, 48-55, 2003.

[2] Tannock, G.W.: A Fresh Look at the Intestinal Microflora, ed. By Tannock, G.W., Probiotics: a critical review. Norfolk, Horizon Scientific Press, pp. 5-14, 1999.

[3] Penders, J., Thijs, C., Vink, C., Stelma, F.F., Snijders, B., Kummeling, I., van den Brandt, P.A., and Stobberingh, E.E.: Factors influencing the composition of the intestinal microbiota in early infancy. Pediatrics, 118, 511-521, 2006.

[4] Gdalevich, M., Mimouni, D., David, M., and Mimouni, M.: Breast-feeding and the onset of atopic dermatitis in childhood: a systematic review and meta-analysis of prospective studies. J. Am. Acad. Dermatol., 45, 520-527, 2001.

[5] Pettigrew, M.M., Khodaee, M., Gillespie, B., Schwartz, K., Bobo, J.K., and Foxman, B.: Duration of breastfeeding, daycare and physician visits among infants 6 months and younger. Ann. Epidemiol., 13, 431-435, 2003.

[6] Zarban, A., Taheri, F., and Chahkandi, T.: Antioxidant and radical scavenging activity of human colostrum, transitional and mature milk. J. Clin. Biochem. Nutr., 45, 150-154, 2009.

[7] Ezaki, S., Ito, T., Suzuki, K., and Tamura, M.: Association between total antioxidant capacity in breast milk and postnatal age in days in premature infants. J. Clin. Biochem. Nutr., 42, 133-137, 2008.

[8] Benno, Y. and Mitsuoka, T.: Development of intestinal microflora in humans and animals. Bifidobact. Microflora, 5, 13-25, 1986.

[9] Wall, R., Hussey, S.G., Ryan, C.A., O’Neill, M., Fitzgerald, G., Stanton, C., and Ross, R.P.: Presence of two Lactobacillus and Bifidobacterium probiotic strains in the neonatal ileum. ISME J., 2, 83-91, 2008.

[10] Harmsen, H.J., Wildeboer-Veloo, A.C., Raangs, G.C., Wagendorp, A.A., Klijn, N., Bindels, J.G., and Welling, G.W.: Analysis of intestinal flora development in breast-fed and formula-fed infants by using molecular identification and detection methods. J. Pediatr. Gastroenterol. Nutr., 30, 6167, 2000.

[11] Boehm, G., Lidestri, M., Cassetta, P., Jelinek, J., Knol, J., Negretti, F., Stahl, B., and Marini, A.: Effect of increasing number of intestinal Bifidobacteria on the presence of clinically relevant pathogens. J. Pediatr. Gastroenterol. Nutr., 36, $578,2003$.

[12] Knol, J., Steenbakkers, G., Van der Linde, E., Gross, S., Helm, K., Klarczyk, M., Schöepfer, H., and Kafka, C.: Bifidobacterial species that are present in breast fed infants are stimulated in formula fed infants by changing to a formula containing prebiotics. J. Pediatr. Gastroenterol. Nutr., 34, 477, 2002.

[13] Gibson, G.R. and Roberfroid, M.B.: Dietary modulation of the human colonic microbiota: introducing the concept of prebiotics. J. Nutr., 125, 1401-1412, 1995.

[14] Roberfroid, M.B.: Prebiotics: preferential substrates for specific germs. Am. J. Clin. Nutr., 73 Suppl., 406S-409S, 2001. 
[15] Gudiel-Urbano, M. and Goñi, I.: Human milk oligosaccharides. The rule in the health and development of the infants. Arch. Latinoam. Nutr., 51, 332-339, 2001.

[16] Roberfroid, M.: Inulin-type fructans and infant formulas, in Inulin-type Fructans, Functional Food Ingredients, eds. By Wolinsky, I., and Hickson, J.F., Boca Raton, CRC Press LLC, pp. 344-345, 2005.

[17] Coussement, P.A.: Inulin and oligofructose: safe intakes and legal status. J. Nutr., 129 Suppl., 1412S-1417S, 1999.

[18] Meyer, D. and Stasse-Wolthuis, M.: The bifidogenic effect of inulin and oligofructose and its consequences for gut health. Eur. J. Clin. Nutr., 63, 1277-1289, 2009.

[19] Ramirez-Farias, C., Slezak, K., Fuller, Z., Duncan, A., Holtrop, G., and Louis, P.: Effect of inulin on the human gut microbiota: stimulation of Bifidobacterium adolescentis and Faecalibacterium prausnitzii. Br. J. Nutr., 101, 541-550, 2009.

[20] K.W. Yap, W., Mohamed, S., Husni Jamal, M., Diederick, M., and A. Manap, Y.: Changes in infants faecal characteristics and microbiota by inulin supplementation. J. Clin. Biochem. Nutr., 43, 159-166, 2008.

[21] Kelly, G.: Inulin-type prebiotics-a review: part 1. Altern. Med. Rev., 13, 315-329, 2008.

[22] Kelly, G.: Inulin-type prebiotics-a review: part 2. Altern. Med. Rev., 14, 35-55, 2009.

[23] Nebra, Y. and Blanch, A.R.: A new selective medium for Bifidobacterium spp. Appl. Environ. Microbiol., 65, 51735176, 1999.

[24] Rogosa, M., Mitchell, J.A., and Wiseman, R.F.: A selective medium for the isolation and enumeration of oral and fecal lactobacilli. J. Bacteriol., 62, 132-133, 1951.

[25] Despopoulos, A. and Silbernagl, S.: Color Atlas of Physiology, 5th Edn. Stuttgart, Georg Thieme Verlag, pp. 226-233, 2003.

[26] General Committee of Revision.: The United States Pharmacopeia, 22nd Revision (USP XXII). Rockville (MD), The United states Pharmacopeial Convention, pp. 1007, 1989.

[27] Collins, C.H., Lyne, P.M., Grange, J.M., and Falkinham, J.O.: Collins and Lyne's Microbiological Methods, 8th Edn. Hodder Arnold, London, pp. 144, 2004.

[28] Gojgic-Cvijovic, G. and Vrvic, M.M.: Practical Microbial Chemistry, 1st Edn. Belgrade, University of Belgrade, pp. 107-108, 2003 (In Serbian).

[29] Manual, Acetic acid, UV Method, AT 2654, Randox, Crumlin, Revised 15/03/99.

[30] Manual, L-Lactic acid, UV Method, LC 2653Randox, Crumlin, Revised 06/08/99.

[31] De Vries, W. and Stouthamer, A.: Fermentation of glucose, lactose, galactose, mannitol and xylose by bifidobacteria. $J$. Bacteriol., 96, 472-478, 1968.

[32] Adams, M.R. and Hall, C.J.: Growth inhibition of food-borne pathogens by lactic acid and acetic acid and their mixture. Int. J. Food Sci. Technol., 23, 287-292, 1988.

[33] Gibson, G.R. and Wang, X.: Regulatory effects of bifidobacteria on the growth of other colonic bacteria. J. Appl. Bacteriol., 77, 412-420, 1994.

[34] Benno, Y., Sawada, K., and Mitsuoka, T.: The intestinal microflora of infants: composition of fecal flora in breast-fed and bottle-fed infants. Microbiol. Immunol., 28, 975-986, 1984.

[35] Quinlan, P.T., Lockton, S., Irwin, J., and Lucas, A.L.: The relationship between stool hardness and stool composition in breast- and formula-fed infants. J. Pediatr. Gastroenterol. Nutr., 20, 81-90, 1995. 